Mathematical Research Letters 4, 143-156 (1997)

\title{
ESTIMATES FOR THE BENNEQUIN NUMBER OF LEGENDRIAN LINKS FROM STATE MODELS FOR KNOT POLYNOMIALS
}

\author{
SERGE TABACHNikov
}

\section{Introduction}

A contact structure in a 3-dimensional manifold is a completely nonintegrable 2-dimensional distribution: if the distribution is the kernel of a (locally defined) 1 -form $\lambda$ then $\lambda \wedge d \lambda \neq 0$ everywhere. The standard contact structure in 3 -space, arising from the identification of $\mathbb{R}^{3}$ with the space of 1 -jets of functions on the line, is given by the contact form $\lambda=d z-y d x$; here $x$ is a point on the line, $z$ is the value of a function and $y$ the value of the derivative at $x$. The same formula defines the standard contact structure in the space of 1-jets of functions on the circle ( $x$ being the cyclic coordinate), the space which is topologically the solid torus. In this paper we will be concerned only with these two contact manifolds.

In a contact 3-dimensional manifold one considers two classes of knots and links: the transverse and the Legendrian ones. The former are everywhere transverse to the contact distribution, and the latter are everywhere tangent to it. Every topological isotopy class of knots in a contact 3 -fold contains a transverse and a Legendrian knot. The main problem of contact knot theory (which has made, so far, only a few first steps) is to classify, up to contact isotopies, transverse and Legendrian knots within each topological isotopy class. In particular, one would like to have specifically contact invariants of transverse and Legendrian knots and links.

One such invariant is easily defined. Legendrian and transverse knots in the standard contact space have natural framings given by the vector fields $\partial_{z}$ and $\partial_{y}$, respectively. The corresponding self-linking number is called the Bennequin number; it is denoted by $\beta(K)$ where $K$ is a Legendrian or a transverse knot $^{1}$. Similarly one defines the Bennequin number of an oriented Legendrian or transverse link.

The study of knots in contact 3-dimensional manifolds was put forward by the seminal paper by D. Bennequin [Be]. One of the main results of this paper

Received May 28, 1996.

Research was supported in part by NSF grant DMS-9402732.

${ }^{1}$ Another simple invariant of a Legendrian knot in the standard contact space is the winding number of its projection to the $(x, y)$-plane, called the Maslov number. There are no other finite order contact invariants (which are not topological invariants) of transverse and Legendrian knots in $J^{1} \mathbb{R}^{1}$ and $J^{1} \mathbb{S}^{1}-$ see $[\mathrm{F}-\mathrm{T}]$, [Go], [Hi]. 
is that for every transverse and Legendrian knot in the standard contact space its Bennequin number is less than twice its genus. In particular, the Bennequin number of a topologically unknotted Legendrian knot is always negative. This remarkable inequality is specific to the standard contact structure, and it fails for other, previously known, contact structures in $\mathbb{R}^{3}$. A consequence is the existence of exotic, so called, overtwisted contact structures (see [El 1]).

The Bennequin's inequality has two shortcomings. First, the genus of a knot, in general, is not computable from knot diagram. Secondly, the genus is insensitive to mirroring, and Bennequin's inequality gives the same estimate for a knot and its mirror image. This makes Bennequin's inequality far from being optimal. The problem of finding a better upper bound for the Bennequin numbers of Legendrian and transverse knots and links within a fixed topological isotopy class became popular in contact topology since Bennequin's paper (see [El 2] and [Ki], problem 1.87).

New estimates for the Bennequin number became available with the advent of "quantum topology". Soon after the introduction of the Homfly polynomial J. Franks and R. Williams $[\mathrm{F}-\mathrm{W}]$ and H. Morton [Mo] found an upper bound for the difference between the algebraic crossing number $c$ and the number of strings $n$ of a closed braid in terms of the Homfly polynomial of the corresponding link. Combined with Bennequin's results that every transverse knot $K$ in the standard contact space is contact isotopic to a closed braid and that $\beta(K)=c-n$, one obtains an upper bound for the Bennequin number of a transverse and of a Legendrian knot in terms of its Homfly polynomial ${ }^{2}$.

These new estimates for the Bennequin number remained unnoticed in the literature for about 10 years until $[\mathrm{F}-\mathrm{T}]$ (probably due to lack of communication between contact topologists and knot theorists, another case of "missed opportunities"). Another result of $[\mathrm{F}-\mathrm{T}]$ is a similar upper bound for the Bennequin number of a Legendrian link in the standard contact space in terms of its Kauffman polynomial ${ }^{2}$.

For example, the Bennequin inequality gives $\beta(K) \leq 1$ for every Legendrian realization $K$ of the right- and left-handed trefoil knots. The new upper bounds for $\beta(K)$ are 1 and -6 , respectively, and these estimates are the best possible (see Theorem 2.1 below, [F-T] and also [Ka]). Interestingly, the Kauffman polynomial appears to be better in this game: the Homfly gives the upper bound of -5 instead of -6 .

The estimates for the Bennequin numbers of Legendrian links in terms of knot polynomials were extended in $[\mathrm{C}-\mathrm{G}]$ to the space $J^{1} \mathbb{S}^{1}$. The proofs proceed (implicitly in $[\mathrm{F}-\mathrm{T}]$ and explicitly in $[\mathrm{C}-\mathrm{G}]$ ) by rather involved induction in the number of double points of a link diagram, using the skein relations for the knot polynomials. On the other hand, Yang-Baxter state models are available for knot polynomials ([Tu 1,2]). In this paper we deduce the Bennequin number

\footnotetext{
${ }^{2}$ There are many versions of knot polynomials; the ones used in this paper are defined in Section 2. The formulations of the inequalities for the Bennequin numbers of Legendrian links in the standard contact space in terms of knot polynomials are given in Theorem 2.1 below.
} 
estimates directly from the state models. We mainly consider the standard contact 3-space, briefly indicating the necessary changes in the solid torus case in the last section of the paper.

\section{Setting the scene: Legendrian link diagrams}

Consider the two projections of the contact $(x, y, z)$-space: on the $(x, y)$-plane and on the $(x, z)$-plane.

The $(x, y)$-projection of a Legendrian knot is an immersed curve; since $d z=$ $y d x$ along a Legendrian curve, this immersed curve bounds zero area. Likewise the $(x, y)$-projection of a Legendrian knot may have a kink shown in Fig. 1 on the left but not the opposite kink shown on the right.
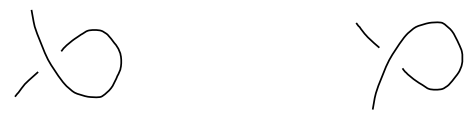

Figure 1: Possible and impossible kinks

The natural framing of Legendrian links is the blackboard framing in this projection, and the Bennequin number equals the writhe, i.e., the algebraic sum of double points - see Fig. 2.

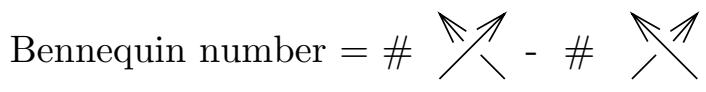

Figure 2: Bennequin number from the $(x, y)$-projection

The $(x, z)$-projection of a Legendrian curve is called its front. A front does not have vertical tangents; generically, its only singularities are transverse double points and semicubical cusps. Since $y=d z / d x$ along a Legendrian curve, the missing $y$ coordinate is the slope of the front. Therefore the front of a Legendrian link is free from selftangencies, and, at a double point, the branch with a greater slope is higher along the $y$ axis.

The Bennequin number of a Legendrian link is expressed in terms of the double points and cusps of its front - see Fig. 3.

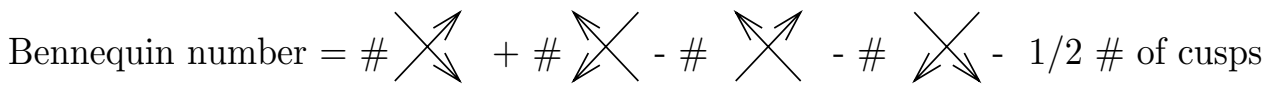

Figure 3: Bennequin number from the $(x, z)$-projection

For example, $\beta=-2$ for the front in Fig. 4 .

Figure 5 shows the correspondence between the $(x, z)$ - and $(x, y)$-projections.

Two Legendrian links are Legendrian isotopic if and only if their fronts are related by a sequence of the Legendrian versions of Reidemeister moves shown in Fig. 6 (see $[\mathrm{Sw}]$ ). 

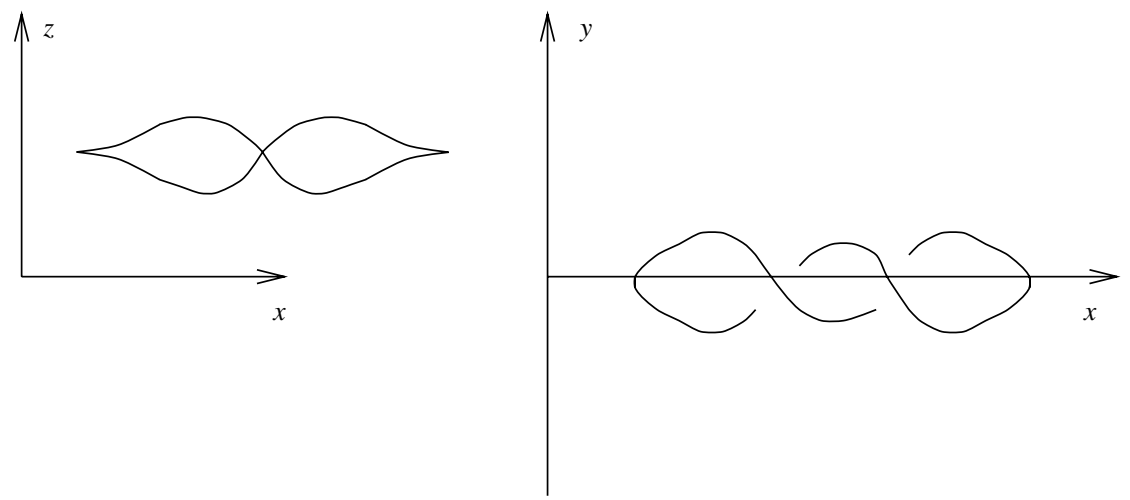

Figure 4: Two projections of a Legendrian (un)knot

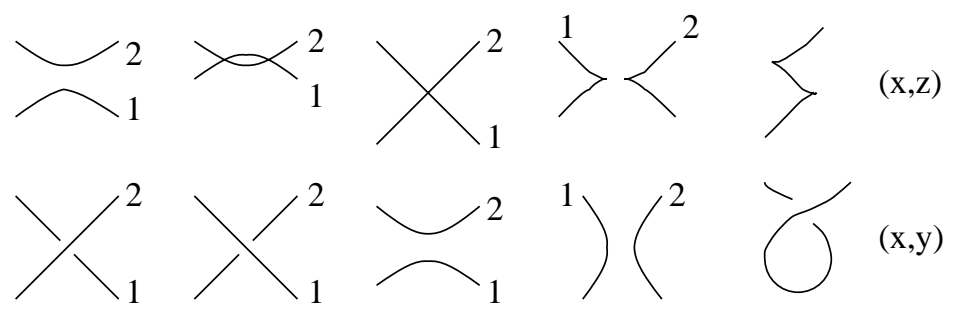

Figure 5: The correspondence between the two projections

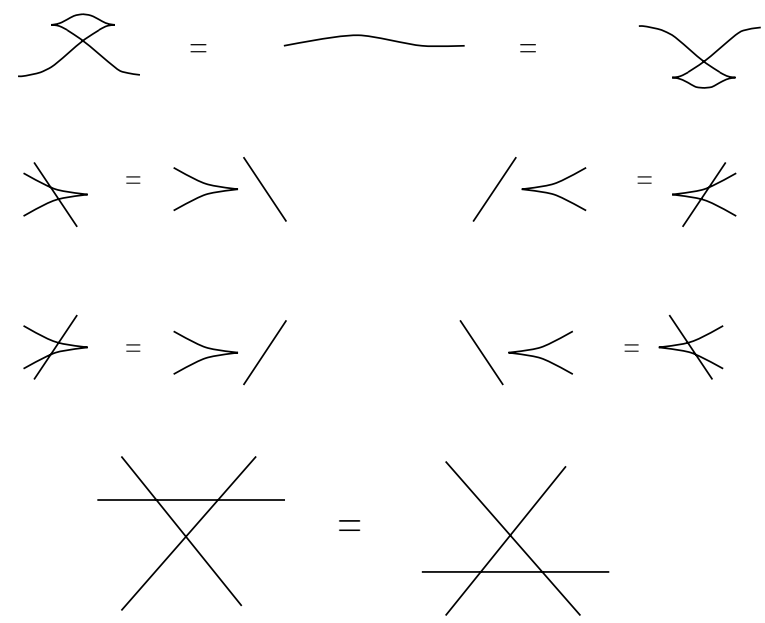

Figure 6: Legendrian Reidemeister moves

We consider the following versions of the Homfly and Kauffman polynomials (slightly different from the ones in $[\mathrm{F}-\mathrm{T}]$ ), described in terms of the $(x, y)-$ projection.

The framed Homfly polynomial $F_{L}(x, y)$ is a Laurent polynomial in $x, y^{3}$ depending on a link $L$ which satisfies the skein relations shown in Fig. 7 (here and

\footnotetext{
${ }^{3}$ Surely these variables have nothing to do with the coordinates in 3-space.
} 
in further skein relations we omit the symbol for the polynomial; it is understood that $F$ takes equal values on the right and the left hand sides).

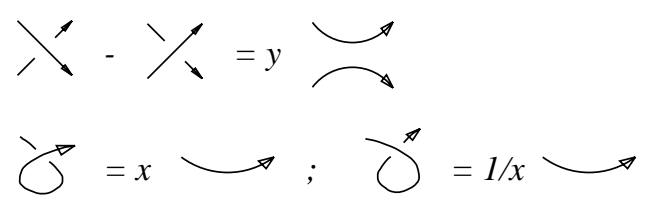

Figure 7: Skein relations for the Homfly polynomial

In addition, $F_{L_{1} \cup L_{2}}=F_{L_{1}} F_{L_{2}}$, where $L_{1} \cup L_{2}$ is the disjoint union of the links $L_{1}$ and $L_{2}$. The Homfly polynomial is $\bar{F}(x, y)=x^{w} F(x, y)$, where $w$ is the writhe.

Likewise, the framed Kauffman polynomial $K_{L}(x, y)$ for nonoriented links satisfies the skein relations shown in Fig. 8.

$$
\begin{aligned}
& \backslash-Y=y)(-y \succsim \\
& \gamma^{\prime}=x \smile ; \delta^{\prime}=1 / x \smile
\end{aligned}
$$

Figure 8: Skein relations for the Kauffman polynomial

In addition, $K_{L_{1} \cup L_{2}}=K_{L_{1}} K_{L_{2}}$. The Kauffman polynomial for oriented links is $\bar{K}(x, y)=x^{w} K(x, y)$. The polynomials $\bar{F}$ and $\bar{K}$ are topological isotopy invariants of links.

Following $[\mathrm{C}-\mathrm{G}]$ one expresses the skein relations for the framed polynomials of Legendrian links in terms of their fronts. In view of Fig. 5, the Homfly polynomial $F$ satisfies the equations in Fig. 9 which will be referred to as front skein relations.

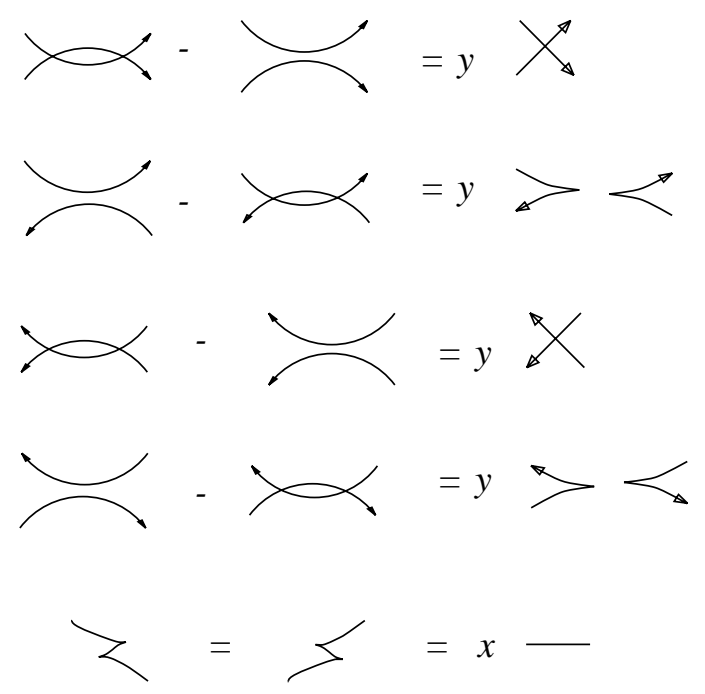

Figure 9: Front skein relations for the Homfly polynomial 
The front skein relations for the Kauffman polynomial $K$ are shown in Fig. 10 .

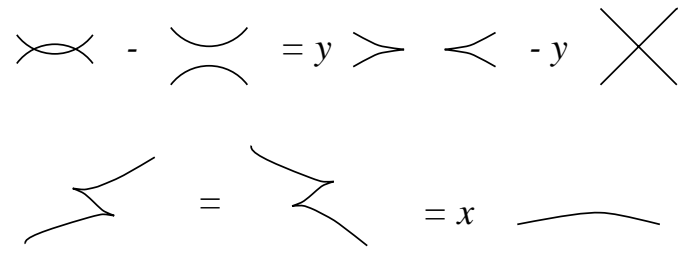

Figure 10: Front skein relations for the Kauffman

In addition, $F$ and $K$ are invariant under the Legendrian Reidemeister moves and

$$
F_{L_{1} \cup L_{2}}=F_{L_{1}} F_{L_{2}}, \quad K_{L_{1} \cup L_{2}}=K_{L_{1}} K_{L_{2}} .
$$

As an example, the equalities for the Kauffman polynomial in Fig. 11 follow from its Legendrian isotopy invariance and the skein relations.

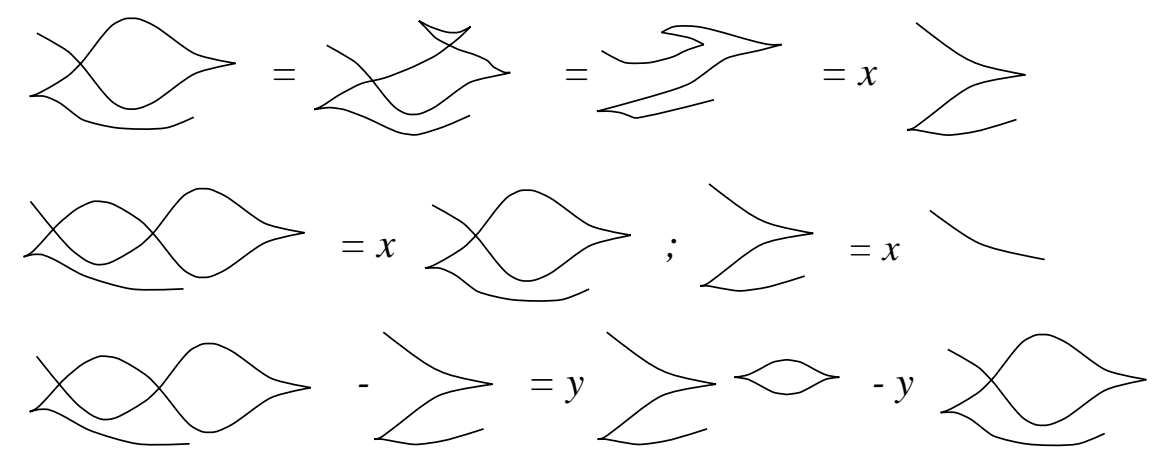

Figure 11: Corollaries from the skein relations

It follows that $K$ takes the value $x+\left(x^{2}-1\right) / y$ on the simplest front, the "flying saucer" (see Fig. 12). The value of $F$ on this front is $\left(1-x^{2}\right) / y$ (as the reader will easily check).

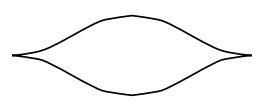

Figure 12: Flying saucer lows.

The inequalities for the Bennequin number from $[\mathrm{F}-\mathrm{T}]$ and $[\mathrm{C}-\mathrm{G}]$ are as fol-

Theorem 2.1. The Bennequin number of a Legendrian link $L$ in the standard contact space does not exceed the minimum of the two numbers: the least degree in $x$ of the Homfly polynomial $\bar{F}_{L}$, and that of the Kauffman polynomial $\bar{K}_{L}$. 
Equivalently, the framed polynomials $F_{L}$ and $K_{L}$ do not contain negative powers of the variable $x$.

The equivalence of the two statements follows from the fact that the Bennequin number is the writhe in the $(x, y)$-projection.

Remarks 1. It follows from Theorem 2.1 that there exists the 1-variable Legendrian link polynomials obtained from $F(x, y)$ and $K(x, y)$ by setting $x=0$. This does not seem to have a counterpart for topological links.

2. Both polynomials $F$ and $K$ take equal values on fronts, symmetric with respect to the $x$ axis. The corresponding contactomorphism of 3 -space

$$
T:(x, y, z) \rightarrow(x,-y,-z)
$$

is topologically but not contactly isotopic to identity ( $T$ changes the sign of the contact 1-form). No nontrivial invariants ${ }^{4}$ are known, at least to the author, which can distinguish between Legendrian links $L$ and $T(L)$.

\section{Uniqueness of the polynomials $F$ and $K$}

In this section we show that the front skein relations determine the Homfly and Kauffman polynomials unambiguously. This result is proved in $[\mathrm{C}-\mathrm{G}]$ in quite a different way.

Theorem 3.1. The front skein relations along with the Legendrian Reidemeister moves invariance uniquely determine the Laurent polynomials $F$ and $K$ on all fronts of Legendrian links.

Proof. Consider the Homfly polynomial, the case of the Kauffman one being completely analogous. Our argument is an adaptation of the standard proof of the fact that skein relations uniquely determine knot polynomials (the existence is quite a different, and harder, matter!)

Let $F$ satisfy the Homfly front skein relations. A double point free front is a disjoint union of "flying saucers" with a number of "zigzags" incerted (see Fig. 13). Therefore, as was above mentioned, the value of $F$ on such a front is determined by the skein relations.

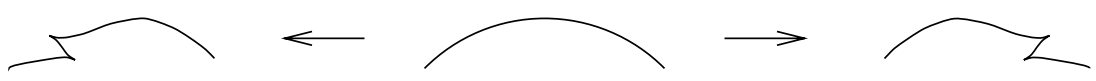

Figure 13: Inserting a zigzag into a front

Given a front $L$ with $N$ double points consider it as a link diagram (whose every double point is of the type shown in Fig. 14 on the left). One may trade some overcrossings for undercrossings to obtain a link diagram of a topologically

\footnotetext{
${ }^{4}$ Except for the Maslov number which is mentioned below.
} 


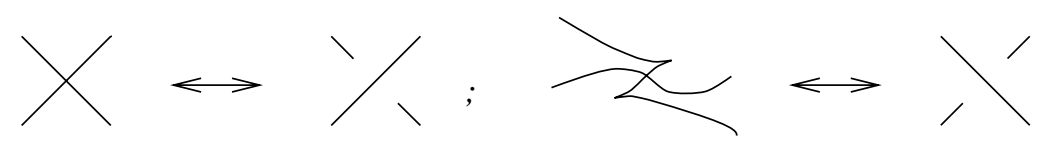

Figure 14: Front versions of crossing changes

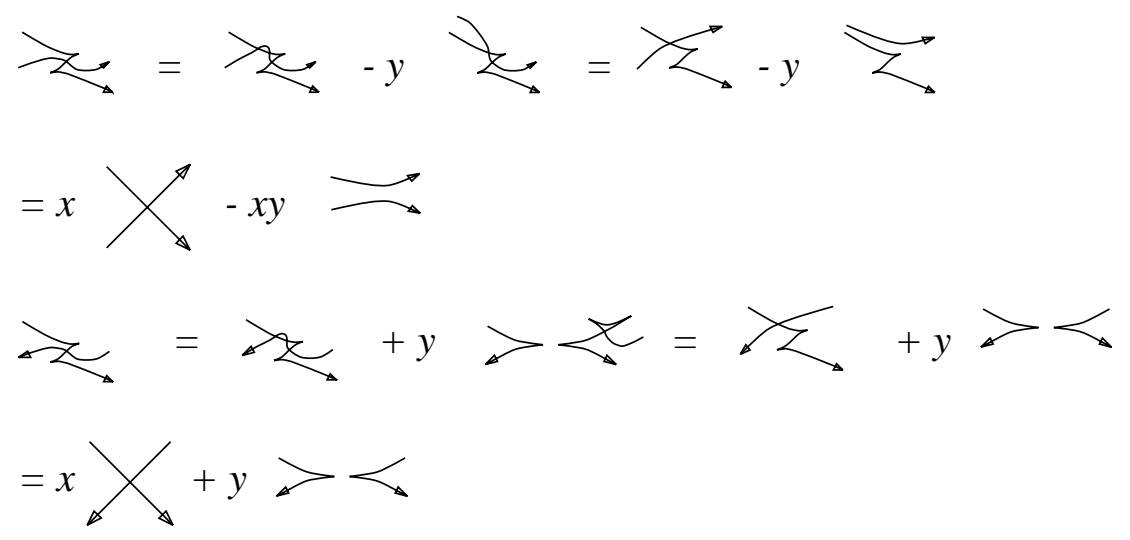

Figure 15: More corollaries from the skein relations

trivial link. The front versions of the crossing change is shown in Fig. 14; note that the two front fragments are not Legendrian equivalent.

The front skein relations and the Legendrian isotopy invariance imply the relations shown in Fig. 15 and the other two similar formulas with other orientations of the branches.

Thus, modulo the values of $F$ on fronts with fewer than $N$ double points, the computation of $F(L)$ reduces to that of $F\left(L_{0}\right)$ where $L_{0}$ is a front of a topologically trivial Legendrian link. That is, $L_{0}$ is topologically isotopic to a Legendrian link with a double point free front.

Next we make use of the following lemma from [F-T] (see also [El 1]):

Lemma 3.2. If two Legendrian links are topologically isotopic then they become Legendrian isotopic after incerting a sufficient number of zigzags into their fronts.

Incerting a zigzag into a front amounts to multiplying $F$ by $x$. Thus the value of $F\left(L_{0}\right)$ is uniquely determined. The proof of the theorem is completed by induction in the number of double points $N$.

\section{State models for polynomials $F$ and $K$}

We modify the state models for the Homfly and Kauffman polynomials from $[\mathrm{Tu}$ 1,2]. These models come from the solutions of the quantum Yang-Baxter equation, associated with the classical Lie algebras of series $A$ and $D$, respectively.

Consider a generic front $L$ as a graph whose vertices are the double points and cusps of $L$. Given a finite set (of colors) $C$, a state of the graph is an assignment 
of an element of $C$ to each edge. To each vertex there corresponds a weight depending on the colors of the edges incident to this vertex. The total weight of a state is the product of the weights of all vertices, and the state sum is the sum of total weights over all colorings.

We specify the set of colors $C$ and the weights below. $C$ will depend on a positive integer $n$, and the weights also on a variable $q$. Thus the state sum will be a function of $q$ and $n$. The state sums for the Homfly and Kauffman polynomials are denoted by $S_{F}(q, n)$ and $S_{K}(q, n)$, respectively.

1) Homfly polynomial.

The set of colors $C=\{1,2, \ldots, n\}$. Set: $y=q-q^{-1}, x=q^{n}$. There are four types of double points shown in Fig. 16 and the corresponding weights are as follows.

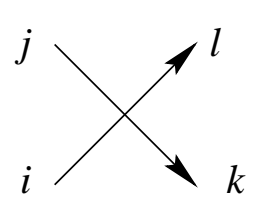

(i)

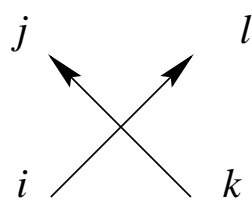

(ii)

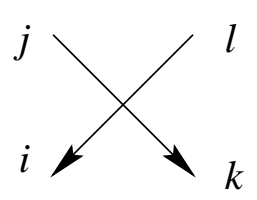

(iii)

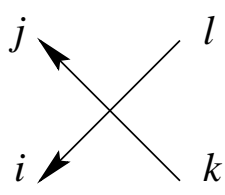

(iv)

Figure 16: Four types of double points for the Homfly polynomial

(i) if $i=j=k=l$ then $w=-q^{-1}$;

if $j=k \neq i=l$ then $w=1$;

if $i=k<j=l$ then $w=y$.

(ii) if $i=j=k=l$ then $w=-q$;

if $j=k \neq i=l$ then $w=1$;

if $i=j<k=l$ then $w=-y q^{k-i}$.

(iii) if $i=j=k=l$ then $w=-q$;

if $j=k \neq i=l$ then $w=1$;

if $i=j>k=l$ then $w=-y q^{i-k}$.

(iv) if $i=j=k=l$ then $w=-q^{-1}$;

if $j=k \neq i=l$ then $w=1$;

if $i=k>j=l$ then $w=y$.

In all other cases the double point weights are equal to zero.

There are four types of cusps shown in Fig. 17 and the weights vanish unless $i=j$; if $i=j$ they equal, respectively,

$$
-q^{n+0.5-i}, \quad q^{n+0.5-i}, \quad q^{i-0.5}, \quad-q^{i-0.5} .
$$

\section{2) Kauffman polynomial.}

The set of colors $C=\{-(2 n-1),-(2 n-3), \ldots,-3,-1,1,3, \ldots,(2 n-1)\}$. Set: $y=q-q^{-1}, x=q^{2 n-1}$. For $i \in C$ denote by $\bar{i}$ the number $i+1$ if $i<0$ and $i-1$ if $i>0$. 

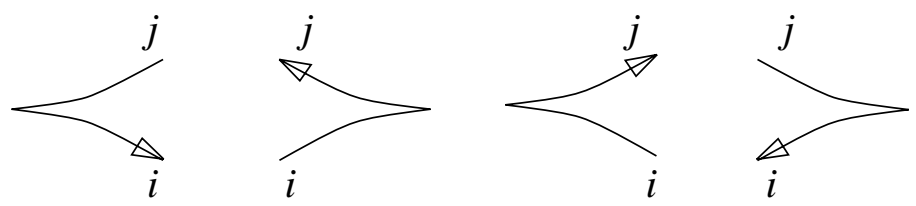

Figure 17: Four types of cusps for the Homfly polynomial

There is only one kind of double point - see Fig. 18, and the weights are:

if $i=j=k=l$ then $w=q^{-1}$;

if $i=l=-j=-k$ then $w=q$;

if $i=l, j=k$ and $i \neq \pm j$ then $w=1$;

if $i=k<j=l$ then $w=-y$;

if $i=-j, k=-l$ and $i<l$ then $w=y q^{(\bar{l}-\bar{i}) / 2}$.

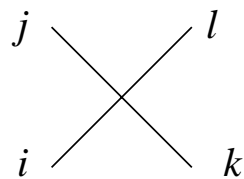

Figure 18: Double point for the Kauffman polynomial

In all other cases the double points weights are equal to zero.

The cusp weights vanish unless $j=-i$; if $j=-i$ then, for both types of cusps, $w=q^{n-(\vec{i}+1) / 2}$. - see Fig. 19 .
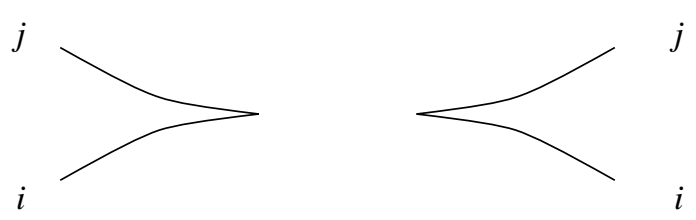

Figure 19: Two types of cusps for the Kauffman polynomial

With this choice of weights the state sums enjoy the following property.

Theorem 4.1. $S_{F}(q, n)$ and $S_{K}(q, n)$ are invariant under the Legendrian Reidemeister moves and satisfy the front skein relations (with the above specified $x$ and $y)$.

We omit the proof which is essentially computational and repeats the argument in $[\mathrm{Tu} \mathrm{1,2]}$; the above weights are slight modifications of the ones from $[\mathrm{Tu}$ $1,2]$.

\section{Proof of Theorem 2.1}

We are ready to prove that the Homfly and Kauffman polynomials $F(x, y)$ and $K(x, y)$ are genuine polynomials in the variable $x$. In a nutshell, the state sums do not contain too great negative powers of $q$ because each weight contributes 
at most $q^{-1}$. On the other hand, a negative power of $x$ would contribute a great negative exponent of $q$ for $n$ great enough.

Proof of Theorem 2.1. Consider $F(x, y)$, the case of $K(x, y)$ being completely analogous.

The state sum $S_{F}(q, n)$ is a Legendrian isotopy invariant and satisfies the front skein relations with $x=q^{n}$ and $y=q-q^{-1}$. It follows from Theorem 3.1 that for every front

$$
S_{F}(q, n)=F\left(q^{n}, q-q^{-1}\right) .
$$

Fix a front $L$; let $F(x, y)$ and $S_{F}(q, n)$ be the corresponding Homfly polynomial and the state sum. Notice that the only negative power of $q$ which appears in the weights of each vertex is $q^{-1}$. Let $v$ be the number of vertices of $L$. It follows that the exponent of each monomial $q^{i}$ in $S_{F}$ satisfies the inequality $i \geq-v$.

Let $m$ be the least degree of $F$ in $y$, and let $u=-\min \{m, 0\} \geq 0$. Set:

$$
F_{1}(x, y)=y^{u} F(x, y)
$$

this is a genuine polynomial in $y$. The exponent of each monomial $q^{i}$ in $F_{1}\left(q^{n}, q-\right.$ $\left.q^{-1}\right)$ satisfies the inequality $i \geq-(u+v)$.

Arguing by contradiction, assume that $F(x, y)$ contains negative powers of $x$. Then

$$
F_{1}(x, y)=\sum_{i=k}^{l} a_{i}(y) x^{i} ; \quad k<0 .
$$

Let $d$ be the top degree of $F_{1}(x, y)$ in $y$, and $e=\operatorname{deg} a_{k}(y)$. The term $a_{k}(y) x^{k}$ contributes the monomials $q^{k n+j}$ to $F_{1}\left(q^{n}, q-q^{-1}\right)$ with $j \leq e$, and the coefficient of $q^{k n+e}$ in $a_{k}(y) x^{k}$ does not vanish.

On the other hand, the exponent of each monomial $q^{j}$ in the terms $a_{i}(y) x^{i}$ with $i>k$ satisfies the inequality $j \geq n(k+1)-d$. Therefore, for sufficiently great $n$ (namely, $n>e+d$ ) the monomial $q^{k n+e}$ does not cancel in $F_{1}\left(q^{n}, q-q^{-1}\right)$. If, in addition, $n>e+u+v$ then $k n+e<-(u+v)$, the latter number being the least posible exponent of the variable $q$ in $F_{1}\left(q^{n}, q-q^{-1}\right)$. This is a desired contradiction.

Remark. The Maslov number $\mu$ of an oriented front is half the difference between the numbers of its descending and ascending cusps; $\mu$ is a Legendrian isotopy invariant. It is proved in $[\mathrm{F}-\mathrm{T}]$ and $[\mathrm{C}-\mathrm{G}]$ that for every front the number $\beta+|\mu|$ is also bounded above by the least degree in $x$ of the corresponding Homfly polynomial $\bar{F}(x, y)$. It is easy to incorporate $\mu$ into the state model (multiplying the cusp weights by $q^{ \pm n / 2}$ ). However the inequality for $\beta+|\mu|$ does not seem to follow the same way, as the one for $\beta$, from the state model.

\section{The space $J^{1} \mathbb{S}^{1}$}

We briefly indicate the modifications of the previous arguments needed in this case. The Homfly and Kauffman polynomials for links in the solid torus were constructed by V. Turaev in [Tu 3]. 
Fronts lie on the cylinder $\mathbb{S}^{1} \times \mathbb{R}^{1}$ rather than in the plane. Each irreducible component of a front contributes an integer, the degree of its projection to $\mathbb{S}^{1}$, in the oriented Homfly case, and a nonnegative integer, the absolute value of the degree of its projection to $\mathbb{S}^{1}$, in the nonoriented Kauffman case. The degree of a front is the sum of these numbers over all components.

Accordingly, the Homfly and Kauffman polynomials depend on extra variables $z_{i}$ with $i$ a nonzero integer in the former and a positive integer in the latter cases. The polynomials $F\left(x, y, z_{i}\right)$ and $K\left(x, y, z_{i}\right)$ satisfy the same front skein relations (involving $x$ and $y$ ), and they take the values $z_{i}$ on the simplest fronts of degrees $i$ shown in Fig. 20 (oriented in the Homfly and not oriented in the Kauffman cases).

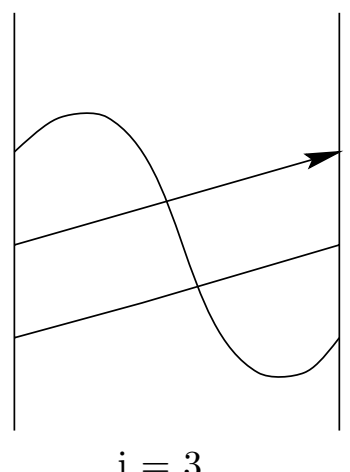

$\mathrm{i}=3$

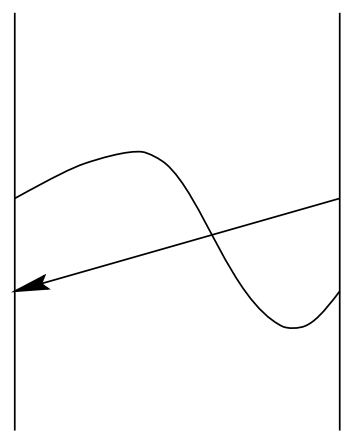

$\mathrm{i}=-2$

Figure 20: Simple fronts of degree $i$

The Bennequin number of a front is given by the same local formula as before, and the polynomials

$$
\bar{F}\left(x, y, z_{i}\right)=x^{\beta} F\left(x, y, z_{i}\right), \quad \bar{K}\left(x, y, z_{i}\right)=x^{\beta} K\left(x, y, z_{i}\right)
$$

are isotopy invariants of links in the solid torus.

The state models are modified as follows. To incorporate the new variables one chooses a vertical line $l$ on the cylinder (say, $x=0$ ). A generic front intersects $l$ off its double points and cusps. These intersections are considered new vertices. Let $t_{1}, \ldots, t_{n}$ be new commuting variables, also commuting with $q$. The weights assigned to the new vertices, shown in Fig. 21, vanish unless $i=j$; if $i=j$ they are:

$$
\text { (i) } w=t_{i} ; \quad \text { (ii) } w=t_{i}^{-1} ; \quad \text { (iii) } w=t_{i}^{\operatorname{sng}(i)}
$$

(cases (i) and (ii) are those of the Homfly and (iii) of the Kauffman polynomials

The state sums become Laurent polynomials in $t_{1}, \ldots, t_{n}, q$ and do not change under the moves in Fig. 22. The variables $x$ and $y$ are related to $q$ and $n$ as before, and $z_{i}$ equals the state sum, corresponding to the front of index $i$ in Fig. 20 . 

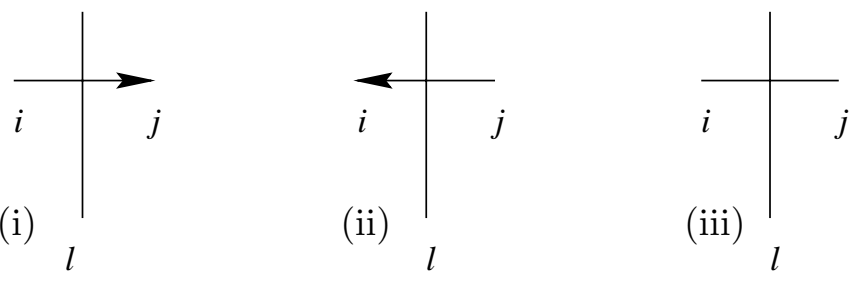

Figure 21: New vertices of a front

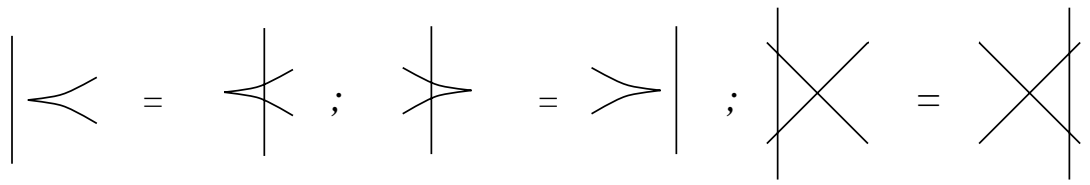

Figure 22: Relative position of a front and the vertical line $l$

After these preparations the previous arguments apply to show that $F\left(x, y, z_{i}\right)$ and $K\left(x, y, z_{i}\right)$ are genuine polynomials in $x$ for every front. This gives an upper bound for the Bennequin number of a Legendrian link in $J^{1} \mathbb{S}^{1}$ within a topological isotopy class.

\section{Acknowledgements}

I am grateful to J. Birman, S. Chmutov and V. Goryunov for numerous stimulating discussions. It is a pleasure to acknowledge the hospitality of the Max-Planck-Institut für Mathematik in Bonn.

\section{References}

[Be] D. Bennequin, Entrelacement et equations de Pfaff, Astérisque 107-108 (1983), 87161.

[C-G] S. Chmutov and V. Goryunov, Polynomial invariants of Legendrian links and plane fronts, Preprint (1996).

[F-W] J. Franks and R. Williams, Braids and the Jones polynomial, Trans. Amer. Math. Soc. 303 (1987), 97-108.

[El 1] Ya. Eliashberg, Classification of overtwisted contact structures on 3-manifolds, Invent. Math. 98 (1989), 623-637.

[El 2] Legendrian and transversal knots in tight contact 3-manifolds, Topol. Methods Modern Math., Publish or Perish, Houston, 1993, pp. 171-193.

[F-T] D. Fuchs and S. Tabachnikov, Invariants of Legendrian and transverse knots in the standard contact space, Topology (to appear).

[Go] V. Goryunov, Vassiliev-type invariants in Arnold's $J^{+}$-theory of plane curves without direct self-tangencies, preprint (1995).

[Hi] J. Hill, Vassiliev-type invariants in $\mathrm{J}^{+}$-theory of planar fronts without dangerous selftangencies, preprint (1995).

[Ka] Y. Kanda, On the Thuston-Bennequin invariant of Legendrian knots and non exactness of the Bennequin inequality, preprint (1995).

[Ki] R. Kirby, Problems in low dimensional manifold theory., preprint (1995).

[Mo] H. Morton, Seifert circles and knot polynomials, Math. Proc. Camb. Phil. Soc. 99 (1986), 107-109. 
[Sw] J. Swiatkowski, On the isotopy of Legendrian knots, Ann. Glob. Anal. Geom. 10 (1992), 195-207.

[Tu 1] V. Turaev, The Yang-Baxter equation and invariants of links, Invent. Math. 92 (1988), $527-553$.

[Tu 2] - Operator invariants of tangles and R-matrices, Math. USSR-Izv 35 (1990), 411-444.

[Tu 3] Conway and Kauffman modules of the solid torus, Zap. Nauchn. Sem. Leningrad. Otdel. Mat. Inst. Steklov. (LOMI) 167 (1988); Issled. Topol. 6, 79-89.

MPim, 26 Gottfried-Claren-Strasse, 53225 Bonn, Germany and Department of Mathematics, University of Arkansas, Fayetteville AR 72701, USA

E-mail address: tabachnikov@mpim-bonn.mpg.de, serge@comp.uark.edu 